\title{
Effect of the Plasmid-DNA Vaccination on Macroscopic and Microscopic Damage Caused by the Experimental Chronic Trypanosoma cruzi Infection in the Canine Model
}

\author{
Olivia Rodríguez-Morales, ${ }^{1}$ Silvia C. Carrillo-Sánchez, ${ }^{1}$ Humberto García-Mendoza, ${ }^{1}$ \\ Alberto Aranda-Fraustro, ${ }^{2}$ Martha A. Ballinas-Verdugo, ${ }^{1}$ Ricardo Alejandre-Aguilar, ${ }^{3}$ \\ José Luis Rosales-Encina, ${ }^{4}$ Maite Vallejo, ${ }^{5}$ and Minerva Arce-Fonseca ${ }^{1}$ \\ ${ }^{1}$ Department of Molecular Biology, Instituto Nacional de Cardiología "Ignacio Chávez", Juan Badiano No. 1, Col. Sección XVI, \\ Tlalpan, 14080 Mexico City, DF, Mexico \\ ${ }^{2}$ Department of Pathological Anatomy, Instituto Nacional de Cardiología "Ignacio Chávez", Juan Badiano No. 1, Col. Sección XVI, \\ Tlalpan, 14080 Mexico City, DF, Mexico \\ ${ }^{3}$ Department of Parasitology, Escuela Nacional de Ciencias Biológicas del I.P.N., Prolongación de Carpio y Plan de Ayala, Col. Sto. \\ Tomás, Miguel Hidalgo, 11340 Mexico City, DF, Mexico \\ ${ }^{4}$ Department of Infectomics and Molecular Pathogenesis, Centro de Investigación y de Estudios Avanzados del I.P.N., \\ Avenida Instituto Politécnico Nacional No. 2508, Col. San Pedro Zacatenco, Gustavo A. Madero, 07360 Mexico City, DF, Mexico \\ ${ }^{5}$ Department of Sociomedical Research, Instituto Nacional de Cardiología "Ignacio Chávez", Juan Badiano No. 1, Col. Sección XVI, \\ Tlalpan, 14080 Mexico City, DF, Mexico
}

Correspondence should be addressed to Minerva Arce-Fonseca; mini_arce@yahoo.com.mx

Received 26 April 2013; Revised 28 July 2013; Accepted 12 August 2013

Academic Editor: Dorothy E. Lewis

Copyright (C) 2013 Olivia Rodríguez-Morales et al. This is an open access article distributed under the Creative Commons Attribution License, which permits unrestricted use, distribution, and reproduction in any medium, provided the original work is properly cited.

\begin{abstract}
The dog is considered the main domestic reservoir for Trypanosoma cruzi infection and a suitable experimental animal model to study the pathological changes during the course of Chagas disease (CD). Vaccine development is one of CD prevention methods to protect people at risk. Two plasmids containing genes encoding a trans-sialidase protein (TcSP) and an amastigote-specific glycoprotein (TcSSP4) were used as DNA vaccines in a canine model. Splenomegaly was not found in either of the recombinant plasmid-immunized groups; however, cardiomegaly was absent in animals immunized only with the plasmid containing the TcSSP4 gene. The inflammation of subendocardial and myocardial tissues was prevented only with the immunization with TcSSP4 gene. In conclusion, the vaccination with these genes has a partial protective effect on the enlargement of splenic and cardiac tissues during the chronic $\mathrm{CD}$ and on microscopic hearth damage, since both plasmids prevented splenomegaly but only one avoided cardiomegaly, and the lesions in heart tissue of dog immunized with plasmid containing the TcSSP4 gene covered only subepicardial tissue.
\end{abstract}

\section{Introduction}

American trypanosomiasis, also known as Chagas disease (CD), is a chronic and potentially fatal disorder, now affecting 10 million people in Latin America [1]. It is one of the major endemic problems in Latin America and ranks as the third most important parasitic disease in the world after malaria and schistosomiasis [2]. An estimate of the number of T. cruzi infections by states in Mexico was made that indicated that the calculated number of potentially affected people is 5.5 million [3]. Several studies describing the seroprevalence in dogs of this country have been conducted and importantly demonstrated a direct correlation with the seropositivity in humans. The seroprevalence in the dog population ranges between $1.6 \%$ and $21 \%$ depending on the geographical region [4-8] suggesting that dogs may be domestic reservoir hosts 
and help to maintain human transmission of T. cruzi when dogs and humans cohabit with vector insects, creating thus a public health problem [9].

Dogs with CD develop diffuse chronic myocarditis with histological and electrocardiographic changes that are also found in humans [10-19]. Prevention of T. cruzi transmission is mainly through the control of vector populations, control of blood transfusion and organ transplantation, and monitoring children from chagasic mothers. However, it is necessary to consider other alternatives, such as the development of vaccines that would be key to improve the control of CD. The development and establishment of regulatory immunization schemes have enabled controlling successfully and even eradicating many diseases worldwide. DNA vaccination represents a promising strategy to attempt to prevent many conditions associated with virulent microorganisms, including obligate and facultative intracellular parasites whose ability to reside within the host cells sustains their resistance to the host's defense mechanisms for longer periods [20].

In previous studies in a murine model, TcSP and TcSSP4 genes immunizations, which were encoding the Trypanosoma cruzi TcSP and TcSSP4 proteins, respectively, were found to be effective in inducing antibodies that were increased after the boost. Protection during the acute phase of the disease was partial: TcSSP4 and TcSP gene-immunized/experimentally infected mice showed a $64 \%$ and a $73 \%$ reduction in parasitemia, respectively. Additionally, survival was 75\% in TcSSP4 gene-immunized/infected mice and 100\% in those immunized with TcSP gene and then infected. These studies demonstrated protection during the chronic phase of the disease as revealed by the histological analysis showing that immunization diminishes cardiac damage caused by the H8 strain of T. cruzi (MHOM/MX/1992/H8 (T. cruzi)) [21-23].

More recently, the clinical and cardiac levels of protection induced by the expression to T. cruzi genes encoding the TcSP and TcSSP 4 proteins in the canine model were reported. Plasmid DNA vaccination with these T. cruzi genes induced a moderate level of protection in the immunized dogs because avoided halted the symptomatic progression to severe heart conduction abnormalities among other effects [24].

Taking advantage of the great similarity between the pathologies developed by chagasic patients and T. cruzi infected dogs, the present study explores the role of the protective effect of TcSP and TcSSP4 gene immunization in Beagle dogs on cardiac and splenic pathological consequences of CD by macro- and microscopic damage findings in order to contribute with new insights about vaccine candidates by reducing infectiousness of the main domestic reservoir of the parasite, as well as by improving the care of companion animals that are increasingly been detected as infected by $T$. cruzi.

\section{Materials and Methods}

2.1. Animals. Thirty-five male and female Beagle dogs aged $16( \pm 1)$ month, and weighing $14.17( \pm 3.8) \mathrm{kg}$ were used; all animals had been immunized against canine distemper, canine parvovirus, coronavirus, hepatitis, leptospirosis, canine infectious tracheobronchitis, and rabies, and intestinal deworming. All of the experimental animals were negative for the standardized enzyme-linked immunosorbent assay (ELISA) [25] used to diagnose CD in dogs prior to the start of the study and were healthy, which confirmed the absence of $\mathrm{CD}$ before any manipulation.

Animal handling followed the established guidelines of the International Guiding Principles for Biomedical Research involving Animals and the Norma Oficial Mexicana: Technical Specifications for the Care and Use of Laboratory Animals [26]. Dogs were euthanized in accordance with the Norma Oficial Mexicana: Humane Slaughter of Domestic and Wild Animals [27]; the experimental protocol was approved by the Bioethics Committee of the Instituto Nacional de Cardiología, Ignacio Chávez.

2.2. Immunization and Challenge. The animals were divided randomly into 5 groups $(n=7)$. The dogs were immunized with $500 \mu \mathrm{g}$ DNA dissolved in $500 \mu \mathrm{L}$ physiological saline solution (SS). The two plasmid constructs ( $\mathrm{pBCSP}$ and pBCSSP 4 containing the TcSP and TcSSP4 genes, resp.) were generated and characterized as described previously [24]. The experimental dogs of each group were immunized with TcSP gene (pBCSP plasmid), TcSSP4 gene (pBCSSP4 plasmid), pBK-CMV (empty cloning vector), or mock-immunized with SS twice at 2-week intervals by intramuscular injection; 15 days after the last immunization, these four groups were infected with a well-characterized Mexican T. cruzi Ninoa strain (MHOM/MX/1994/Ninoa (T. cruzi I)) [23, 28], by intraperitoneal injection with 500,000 metacyclic trypomastigotes per animal that were obtained from urine and feces of triatomines and resuspended in SS. The animals in the healthy control group were not subjected to any experimental procedure. The experimental T. cruzi infection was confirmed microscopically (parasitemia) in all infected groups by examining freshly isolated blood samples collected every third day observing from 200 to 400 parasites $/ \mathrm{mL}$ as limit of detection intermittently between day 22 and day 55 postinfection. All animals were monitored clinically by general physical examinations and electrocardiographics studies. A serological monitoring (ELISA and indirect immunofluorescence as confirmatory test) was also performed in pBK-CMV plasmid-immunized and SS mock-immunized/infected dogs [24] in order to evaluate the establishment of infection by the presence of antibodies anti-T. cruzi.

2.3. Electrocardiograms (EKGs). Once CD chronic phase had been determined by serology and electrocardiographic studies at six months postinfection [24], the last EKGs (Schiller, AT-1, USA) for each animal were obtained (previous to the euthanasia, at 11 months after inoculation). No chemical restraint was employed. Three bipolar standard leads (I, II, and III), three augmented unipolar limb leads (aVR, aVL, and $\mathrm{aVF})$, and four unipolar precordial thoracic leads $\left(\mathrm{CV}_{5} \mathrm{RL}\right.$, $\mathrm{CV}_{6} \mathrm{LL}, \mathrm{CV}_{6} \mathrm{LU}$, and $\mathrm{V}_{10}$ ) were recorded. The voltage was standardized at $1 \mathrm{mV} / \mathrm{cm}$, a paper speed of $50 \mathrm{~mm} / \mathrm{s}$ was used, and electrocardiographic tracings were analyzed according to published data in canine and feline cardiology $[29,30]$. 
TABLE 1: Number of animals with presence of major cardiac anomalies (by EKG) in DNA-immunized dogs with chronic experimental Chagas disease.

\begin{tabular}{lll}
\hline Group & Dogs/n(\%) & Suggested pathological conditions by EKG features found [29, 30] \\
\hline TcSP gene (pBCSP plasmid) & $2 / 7(29 \%)$ & AV block \\
& $1 / 7(14 \%)$ & Left ventricular enlargement \\
TcSSP4 gene (pBCSSP4 & $1 / 7(14 \%)$ & MIMI \\
plasmid) & $1 / 7(14 \%)$ & Second-degree AV block \\
pBK-CMV (empty plasmid) & $6 / 7(86 \%)$ & Most often abnormal when found on serial EKG and combined with other disturbances \\
& $4 / 7(57 \%)$ & Left ventricle enlargement \\
& $1 / 7(14 \%)$ & Right BBB \\
& $3 / 7(43 \%)$ & Most often abnormal if it is found on serial EKG and combinedwith other disturbances \\
SS (mock-immunized) & $1 / 7(14 \%)$ & VPC \\
& $2 / 7(29 \%)$ & Pericardial effusion \\
& $2 / 7(29 \%)$ & Myocardial infarction and/or pericarditis and right ventricular enlargement \\
\hline
\end{tabular}

EKG: electrocardiogram.

AV: atrioventricular.

MIMI: microscopic intramural myocardial infarctions.

BBB: bundle branch block.

SS: physiologic saline solution.

VPC: ventricular premature complexes.

2.4. Euthanasia. Dogs were euthanized at chronic phase (11 months after infection) using sodium pentobarbital (Barbithal, Holland Animal Health, Mexico) as a general anesthetic at a dose of $30 \mathrm{mg} / \mathrm{kg}$ applied intravenously, and then a lethal dose of intravenous $15 \%$ potassium chloride was administered.

2.5. Macroscopic Evaluation. Prior to euthanizing, weight (Bascule Inpros S.A. de CV, Mexico) was obtained and the heart and the spleen were collected during necropsy and also were weighed (Scout Pro, Ohaus, Mexico). Cardiomegaly and splenomegaly were evaluated by determining the heart and spleen indices (organ weight/total body weight $\times 100$ ), respectively, and also by inspecting for macroscopic alterations. Cardiomegaly and splenomegaly were considered present in the animals when the organ index was significantly higher than that observed in healthy non-infected animals [31].

2.6. Histology. Several tissues such as spleen, skeletal muscle, esophagus, ileum, colon, and heart muscle were analyzed. Tissues sections were fixed in $10 \%$ buffered formalin solution for $24 \mathrm{~h}$, dehydrated in absolute ethanol, cleared in xylene, and embedded in paraffin for histological examination. Sections $(5 \mu \mathrm{m})$ were stained with hematoxylin and eosin and evaluated by light microscopy (Carl Zeiss, K7, Germany). Three different sites of heart walls from the top, middle, and bottom of the tissue were analyzed: subepicardium, myocardium, and subendocardium. Images were obtained through a Bio-Doc-It Imaging System image analyzer (UVP, LLC, USA). The severity of inflammation in affected tissue sections was scored on a scale from 1 to 4 . A score of 1 indicated one or less foci of inflammatory cells/field (400x); 2 , more than one inflammatory foci/field; 3 , generalized coalescing of foci of inflammation or disseminated inflammation with minimal cell necrosis and retention of tissue integrity, and 4, diffuse inflammation, with severe tissue necrosis, interstitial edema, hemorrhage, and loss of tissue integrity.

2.7. Statistical Analysis. Heart and spleen indices and histological data were analyzed by the Kruskal-Wallis test (SPSS software, version 13.0). Heart and spleen indices data from all vaccinated groups (including the mock-immunized/infected group as positive control for infection) were compared among themselves and between the non-infected group (healthy control). The histological data from all vaccinated groups were compared among themselves and between the mockimmunized/infected group (control for infection). The inflammation 1 to 4 scores were converted to logarithms (base 10) after 1 was added to each score to correct for 0 values [32], and the group means were compared with the statistical test. For all cases, differences were considered as significant when $P<0.05$.

\section{Results}

3.1. DNA Vaccination with T. cruzi Genes Halted the Progression to Severe Heart Electrical Conduction Abnormalities. The survival rate in all experimental groups was $100 \%$. Before the death by euthanasia, the dogs immunized with pBK-CMV empty plasmid or SS mock-immunized exhibited more severely altered electrocardiographic features, and the number of affected dogs was also higher in contrast with the recombinant plasmids-immunized animals (Table 1).

A P-R interval longer (0.14 s) than normal (0.12 s), associated with atrioventricular (AV) block, was present in 2 dogs (29\%) immunized with the TcSP gene. One animal (14\%) vaccinated with the TcSSP4 gene exhibited features that are 


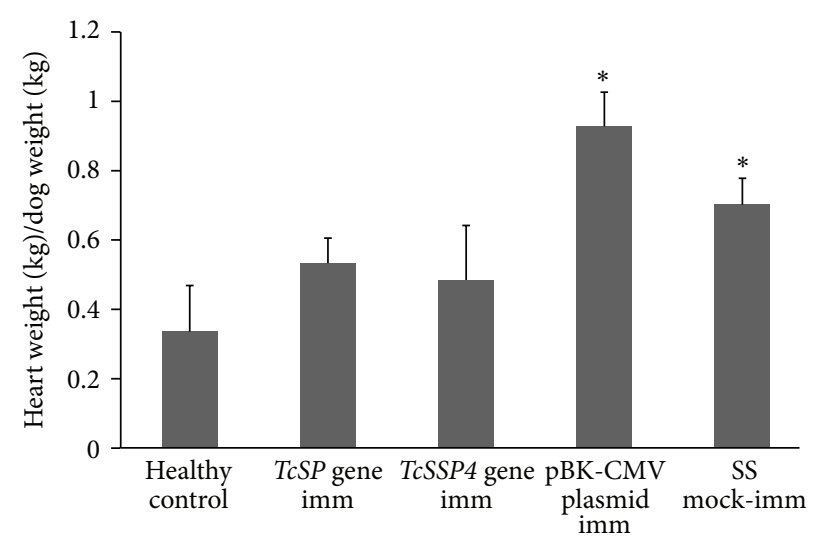

Experimental groups

(a)

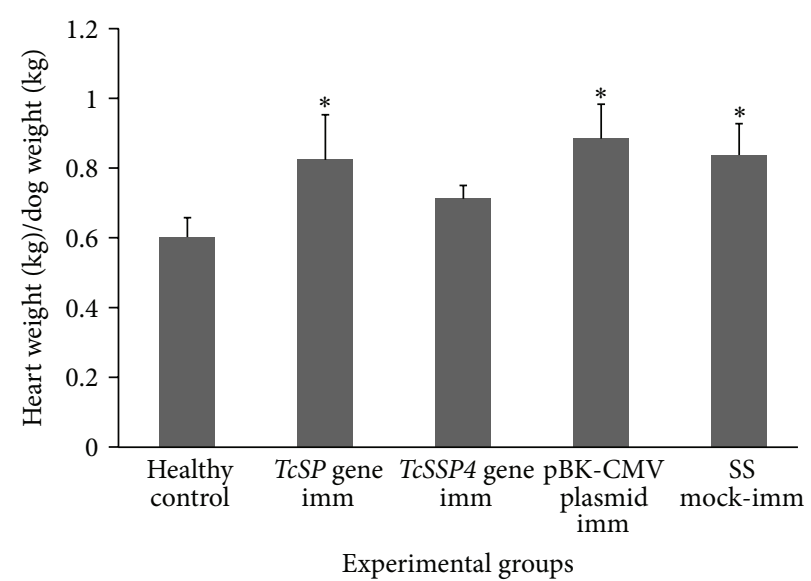

(b)

FIGURE 1: Splenomegaly and cardiomegaly during chronic stage of infection with Trypanosoma cruzi Ninoa strain in Beagle dogs. Splenomegaly and cardiomegaly were calculated by the mean spleen (a) and the mean heart (b) indices, respectively ( \pm S.D.). Differences were considered as significant when $P \leq 0.05$ (Kruskal-Wallis test among immunized/infected groups (including the mock-immunized/infected animals) and healthy non-infected animals was performed). imm = immunization.

consistent with microscopic intramural myocardial infarctions (Table 1). In a previous EKG (6 months postinfection) this dog had shown recordings associated with myocardial infarction and left bundle branch block (BBB) [24].

Reversal in polarity of the $\mathrm{T}$ wave on serial EKGs was present in 6/7 dogs (86\%) of the pBK-CMV empty plasmidimmunized/infected group and in $3 / 7$ dogs (43\%) of the SS mock-immunized/infected group. This abnormality was combined with other disturbances suggesting left ventricle enlargement that could be associated to a dilated form of cardiomyopathy caused by a primary myocardial disease such as CD. Altered T wave, QRS complexes, and S waves suggesting right $\mathrm{BBB}$ were present in one $\operatorname{dog}(14 \%)$ from the $\mathrm{pBK}$ CMV-immunized/infected group (Table 1).

Electrocardiographic features associated with ventricular premature complexes were present in $14 \%$ of the dogs $(1 / 7)$ of the SS mock-immunized/infected group. Additionally, disturbances in $\mathrm{R}$ wave and electrical alternans that suggested pericardial effusion were also exhibited by two dogs (29\%) of this group. Finally, abnormal S-T segment, R wave, and $S$ wave were found in $29 \%$ of the animals (2/7) of the SS mock-immunized/infected group, which suggested myocardial infarction and/or pericarditis and right ventricular enlargement (Table 1).

\subsection{DNA Vaccination with T. cruzi Genes Prevents Spleen but} Not Heart Damage during the Chronic Stage of the Disease. The $\mathrm{pBK}-\mathrm{CMV}$ empty cloning vector-immunized/infected and the SS mock-immunized/infected groups showed splenomegaly (Figure 1(a)) and cardiomegaly (Figure 1(b)) during the chronic phase of the disease as revealed by the mean spleen and heart indices, respectively, as compared with the healthy control group.

DNA vaccination with the TcSSP 4 and TcSP genes prevented splenic tissue damage (Figure 1(a)) during the chronic phase of the infection with $T$. cruzi without differences between them as evidenced by similar spleen index in dogs immunized with each of both genes to that obtained from the healthy non-infected control animals. The dogs immunized with TcSP gene (pBCSP plasmid) and the empty plasmid showed heart indices similar to those of SS mockimmunized/infected group (Figure 1(b)), with a significant difference from the healthy non-infected control group, suggesting cardiomegaly, and without differences between themselves. On the contrary, the heart index of the TcSSP4 gene-(pBCSSP4 plasmid) immunized/infected group was not different from that of the healthy non-infected control animals (Figure 1(b)), suggesting that probably the TcSSP4 gene has a partial protective role on cardiac pathological consequences of $\mathrm{CD}$ that lead to cardiomegaly.

3.3. Other Macroscopic Alterations in Dogs with Chronic $C D$. The lesions found in $71 \%$ of dogs $(5 / 7)$ of the SS mock-immunized/infected group and in $86 \%$ of animals (6/7) immunized with pBK-CMV empty cloning vector were whitish areas in the heart of fibrous consistency, abundant pericardial fluid, and white areas in the spleen (Table 2). One infected dog (14\%) immunized with pBK-CMV plasmid showed ascites, megaesophagus, left ventricular hypertrophy, thinning of the right ventricular wall, and severe tricuspid endocarditis (Table 2). In addition, one dog (14\%) of the TcSSP4 gene-(pBCSSP4 plasmid) immunized/infected group exhibited a heart with adhesions in the trachea and pericardium; splenic thickened walls; and white areas in the spleen and heart (Table 2).

3.4. Histopathologic Analysis. Although several tissues were analyzed (spleen, skeletal muscle, esophagus, ileum, colon and heart muscle), the microscopic findings of T. cruziinduced damage in DNA immunized and infected dogs 
TABLE 2: Other macroscopic alterations in DNA-immunized dogs with chronic experimental Chagas disease.

Macroscopic pathological features

at 11 months after infection

(at the time of euthanizing)

Whitish areas in heart of fibrous consistency

Abundant pericardial fluid

Whitish areas in spleen

Ascites

Megaesophagus

Left ventricular hypertrophy

Thinning of the right ventricular wall

Severe tricuspid endocarditis

Heart with adhesions in trachea and pericardium

Splenic thickened walls

Whitish areas in spleen and heart

$\begin{array}{cl}\text { SS (mock-immunized) and } & 5 / 7(71 \%) \\ \text { pBK-CMV (empty plasmid) } & 6 / 7(86 \%)\end{array}$

pBK-CMV (empty plasmid) $\quad 1 / 7(14 \%)$

TABLE 3: Inflammatory lesion (lymphocyte infiltrates) scores from three different sections of heart tissues of DNA-immunized dogs with chronic experimental Chagas disease.

\begin{tabular}{lcccc}
\hline Site & $\begin{array}{c}\text { TcSP gene } \\
\text { (pBCSP plasmid) }\end{array}$ & $\begin{array}{c}\text { TcSSP4 gene } \\
\text { (pBCSSP4 plasmid) }\end{array}$ & $\begin{array}{c}\text { pBK-CMV } \\
\text { (empty plasmid) }\end{array}$ & $\begin{array}{c}\text { SS } \\
\text { (mock-immunized) }\end{array}$ \\
\hline Subepicardium & $0.800 \pm 0.2^{*}$ & $0.99 \pm 0.4^{*}$ & $1.0714 \pm 0.4^{*}$ & $1.0 \pm 0.0^{*}$ \\
Myocardium & $0.7429 \pm 0.1^{*}$ & $0.5143 \pm 0.1$ & $0.7143 \pm 0.4^{*}$ & $0.9429 \pm 0.4^{*}$ \\
Subendocardium & $0.1429 \pm 0.03$ & $0.2857 \pm 0.04$ & $0.7143 \pm 0.4^{*}$ & $0.8932 .3^{*}$ \\
\hline
\end{tabular}

${ }^{*}$ Differences were considered as significant when $P \leq 0.05$ (Kruskal-Wallis test among immunized/infected and healthy non-infected control animals, whose values obtained were 0.0 , was performed).

were limited exclusively to heart tissue. No evidence of amastigotes' nests was observed in any tissue analyzed of all experimentally infected dogs.

Similar microscopic changes of sections of heart tissue were found in all DNA immunized/infected and SS mock-immunized/infected groups, including inflammatory infiltrates with mononuclear and polymorphonuclear cells and without parasitism in this tissue (Figure 2). Lymphocytes were the predominant cell infiltrates along with a few histiocytes and neutrophils. Alterations related to heart inflammation were identified in $100 \%$ of the dogs immunized with TcSSP4 gene (pBCSSP4 plasmid), pBK-CMV, and inoculated with SS groups, whereas in the TcSP gene(pBCSP plasmid) immunized/infected animals abnormalities were found in $71 \%(5 / 7)$. However, the extent of inflammation of the heart tissue during the chronic phase of infection was reduced in dogs immunized with the recombinant plasmids, as evidenced by the significant differences in the inflammatory lesion scores between subendocardial tissues from dogs immunized with recombinant plasmids and those immunized with the pBK-CMV empty cloning vector or SS mock-immunized (Table 3), as well as between myocardial tissues from TcSSP 4 gene-(pBCSSP 4 plasmid) immunized/infected dogs and from all other groups (Table 3). In immunized/infected groups (including the mock-immunized/infected group as control for infection) with similar inflammatory lesion scores, no significant differences among themselves were found.

\section{Discussion}

In this study cardiomegaly was a condition present in all dogs infected with T. cruzi Ninoa strain as one of the symptoms of heart dysfunction in chronic CD. Experimental studies indicate that different $T$. cruzi laboratory strains exhibit varying degrees of tissue tropism, and the pattern of inflammation in tissues and organs allowed the classification of T. cruzi strains into six discrete typing units (DTUs) according to their genetic background and biodomes. In Mexico, the most of the T. cruzi strains that have been genetically analyzed to date belong to the T. cruzi I group, such as T. cruzi Ninoa strain (MHOM/1994/MX/Ninoa (T. cruzi I)), a reference Mexican strain used in many kinds of studies, which has been classified as a low-virulence strain belonging to biodeme 3 and T. cruzi I group in accordance with these criteria [28, 33, 34]; it can behave pathogenically in the human host, as described in the murine model and now in the canine model [24, 25, 35].

Mortality in the groups of dogs was not observed throughout the experiment, unlike other studies where experimental $T$. cruzi inoculation caused sudden death in some of the infected dogs $[10,11,17,32,36]$; however, some of these authors worked with mongrel dogs and with different number of animal samples, with another T. cruzi strain or morphologic form of the parasite and different amounts of inoculum, or employed different administration routes. Our experimental canine model of CD has already been standardized in accordance with lethal doses 50 assays to determine 


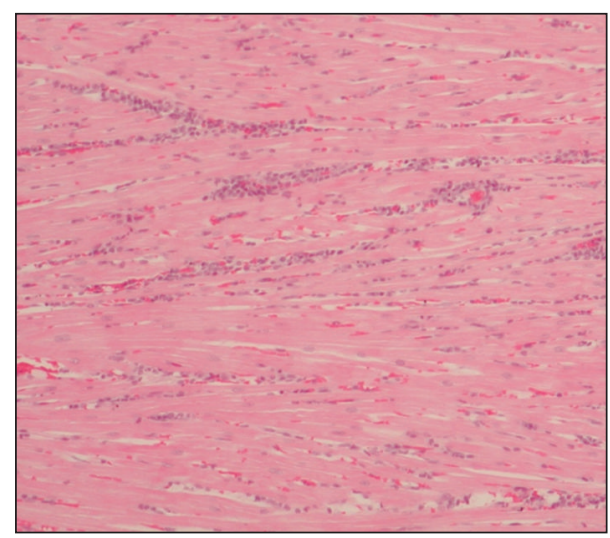

(a)

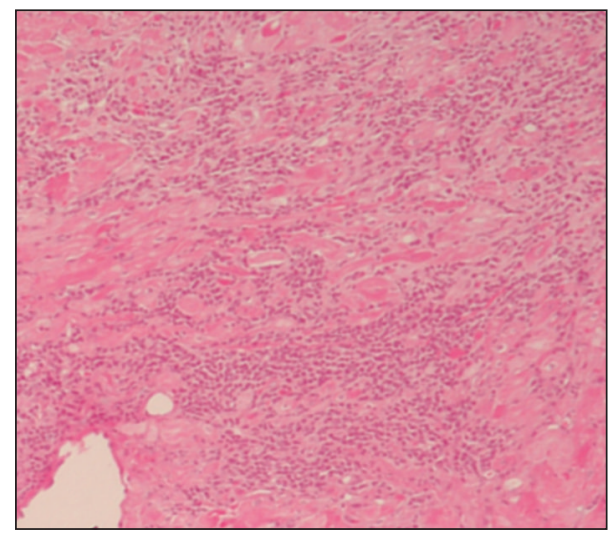

(c)

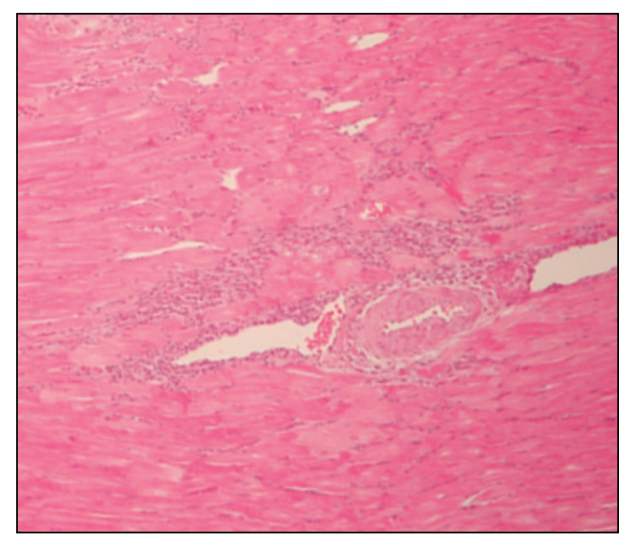

(b)

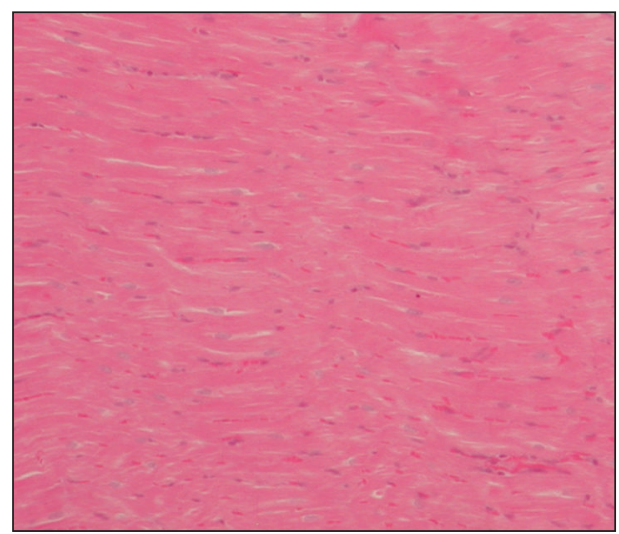

(d)

Figure 2: Histological cardiac sections from Beagle dogs DNA-immunized and infected with Ninoa strain of T. cruzi. Representative micrographs are shown. (a) Score 1 (1 or less foci of inflammatory cells/field) from a dog immunized with TcSSP4 gene (pBCSSP4 plasmid); (b) score 2 (>1 inflammatory foci/field) from a dog immunized with TcSP gene (pBCSP plasmid); (c) score 3 (generalized coalescing of foci of inflammation or disseminated inflammation with minimal cell necrosis and retention of tissue integrity) from a dog mock-immunized with SS, and (d) score 0 (without foci of inflammation) from a healthy/uninfected dog. Hematoxylin and eosin stain; 10x magnification.

an adequate inoculum and achieving the establishment of the acute and chronic phases of CD through the systemic infection (one of the natural routes of infection in dogs, besides of the oral route by ingesting the vector).

Our observations confirmed that DNA-immunization did not abrogate the parasitemia found in all experimental groups during the acute phase of $\mathrm{CD}$; however, the antibodies produced by DNA-immunized dogs mediated the lysis of parasites through the complement system, which had an impact during the acute phase of the illness because parasitemia and parasite load was lower over time in the animals of the vaccinated groups than those nonimmunized dogs [35].

The cardiomegaly, inflammation, and fibrosis were associated to the presence of high in vitro infectivity of T. cruzi strains. Despite that Ninoa T. cruzi is a strain of low virulence, it was possible to find heart and spleen histological changes suggestive of cardiomegaly, myocarditis, and splenomegaly, as reported by other authors $[18,28,31,33,34]$.

Regarding the spleen index, the results show no significant difference in the groups immunized with either TcSP gene or TcSSP4 gene, unlike the group immunized with the empty cloning vector $\mathrm{pBK}-\mathrm{CMV}$, which coincided with splenic indices of SS mock-immunized/infected dogs. This event indicates that infection with the T. cruzi Ninoa strain in the canine model induced splenomegaly in $\mathrm{CD}$ chronic phase similarly to other more virulent strains in humans and dogs $[18,37]$, and the DNA vaccination with parasite TcSP and TCSSP4 genes showed a significant amelioration of splenic pathology, as assessed by the splenic index. This finding correlates directly with splenic tissue damage during the chronic stage of the disease. Parasite TcSP and TcSSP4 genes encode a trans-sialidase and an amastigote specific-protein, which are expressed in all forms of the parasite and in trypomastigote to amastigote transition forms, respectively, stimulating a strong humoral and cellular response during $\mathrm{BALB} / \mathrm{c}$ mice infection and preventing pathological manifestations of chronic $\mathrm{CD}$ $[21,22]$.

Lesions observed in chronic chagasic cardiomyopathy (CCC) frequently produce electrocardiographic alterations and affect cardiac function. The abnormalities recorded in the EKGs agreed with the cardiomegaly found in the infected dogs and the TcSP gene-immunized/infected group, this is 
consistent with reports in studies with Beagle dogs infected with other T. cruzi strains, where mild splenomegalies and severe cardiomegalies were observed in chronic CD [18, 31]. In contrast, the dogs immunized with the TcSSP4 gene at the same dose and then infected exhibited a heart index similar to that of the healthy non-immunized group, and probably TcSSP4, the amastigote specific protein, encoded by the TcSSP4 gene, had a partial protective role during CCC likely because this gene induces immunity against the stagespecific surface antigen present on the intracellular form. Further studies of immunizations with the two plasmids in combination are required to determine if there is a possible synergistic effect.

In some reports that used mongrel dogs for experimental CD cardiomegaly, chronic myocarditis with variable reduction levels of cardiac muscle, fibrosis, and adipose tissue replacement was the most frequent findings $[10,13,14]$.

Other macroscopic findings at necropsy in some dogs are consistent with reports that registered macroscopic and microscopic injuries, such as slight right ventricle dilation and infarcted areas in cardiac tissue, different degrees of lymphoplasmacytic inflammation in myocardium [36], diffuse fibrous cardiopathy [11], chronic focal and discrete myocarditis, whitish thickening in the form of plaque in the right atrium (chronic productive epicarditis), chronic productive perisplenitis [38], fibrosis partially or completely interrupting the path of muscle bundles, cardiomegaly, heart with a globus shape, hydropericardium [13], dilated cardiomyopathy (biventricular dilation), focal and diffused myocarditis, round shaped hearts, and epicardial hemorrhages [19].

No amastigote nests were found in any heart sections obtained from immunized/infected or mock-immunized/ infected dogs with chronic CD; this is consistent with other studies in dogs $[12,13,38]$. Parasites in tissue fragments of dogs, mice, and humans have only been found during the acute stage of CD, but not during the chronic stage $[19,37,39]$; however, effective immunological control of inflammation during the chronic phase of infection resulted in reduced lymphocyte infiltrates by vaccination with the TcSSP 4 gene.

\section{Conclusions}

T. cruzi Ninoa strain infection induces splenomegaly and cardiomegaly, and DNA vaccination with both TcSP and TcSSP4 genes prevents splenic but not cardiac tissue damage during chronic CD. Immunization with the TcSSP4 gene (pBCSSP 4 plasmid) had a partial protective role avoiding cardiomegaly and microscopic heart damage since the dogs of this group showed heart indices similar to those of healthy animals, and the microscopic lesions did not reach myocardial or subendocardial tissues; therefore, DNA vaccination could be an approach to reduce the severity of chronic T. cruzi infection.

\section{Conflict of Interests}

None of the authors has any potential financial conflict of interests related to this paper.

\section{Acknowledgments}

This research was funded by the Instituto de Ciencia $y$ Tecnología del Distrito Federal (ICyTDF) Mexico, Grant no. 236/2010, and by the Consejo Nacional de Ciencia y Tecnología (CONACyT), Mexico, Grant no. 69081 FONSEC SSA/IMSS/ISSSTE. The authors MVZ. Yukie V. Tachika from Facultad de Medicina Veterinaria y Zootecnia of Universidad Nacional Autónoma de México for her invaluable help in EKG interpretation.

\section{References}

[1] World Health Organization, Weekly Epidemiological Record, vol. 87, no. 51/52, pp. 509-526, 2012.

[2] J. A. Marin-Neto and A. Rassi Jr., "Actualización sobre la cardiopatía de la enfermedad de Chagas en el primer centenario de su descubrimiento," Revista Espanola de Cardiologia, vol. 62, no. 11, pp. 1211-1216, 2009.

[3] A. Carabarin-Lima, M. C. González-Vázquez, O. RodríguezMorales et al., "Chagas disease (American trypanosomiasis) in Mexico: an update," Acta Tropica, vol. 127, no. 2, pp. 126-135, 2013.

[4] G. Campos-Valdéz, R. Barrera-Peña, S. R. de la Luz, E. Jiménez-Cardoso, and P. Martínez-Labat, "Anti-Trypanosoma cruzi antibodies in dogs (Canis familiaris) from tlalnepantla municipality, State of Mexico," Bioquimia, vol. 26, no. 1, pp. 1619, 2001.

[5] F. Sosa-Jurado, J. L. Zumaquero-Ríos, P. A. Reyes, A. CruzGarcía, C. Guzmán-Bracho, and V. M. Monteón, "Factores bióticos y abióticos que determinan la seroprevalencia de anticuerpos contra Trypanosoma cruzi en el municipio de Palmar de Bravo, Puebla, México," Salud Pública de México, vol. 46, no. 1, pp. 39-48, 2004.

[6] J. G. Estrada-Franco, V. Bhatia, H. Diaz-Albiter et al., "Human Trypanosoma cruzi infection and seropositivity in dogs, Mexico," Emerging Infectious Diseases, vol. 13, no. 4, pp. 624-630, 2006.

[7] M. Jimenez-Coello, M. Poot-Cob, A. Ortega-Pacheco et al., "American trypanosomiasis in dogs from an urban and rural area of Yucatan, Mexico," Vector-Borne and Zoonotic Diseases, vol. 8, no. 6, pp. 755-761, 2008.

[8] A. Barbabosa-Pliego, H. M. Díaz-Albiter, L. Ochoa-García et al., "Trypanosoma cruzi circulating in the southern region of the State of Mexico (Zumpahuacan) are pathogenic: a dog model," The American Journal of Tropical Medicine and Hygiene, vol. 81, no. 3, pp. 390-395, 2009.

[9] G. B. C. Turriago, G. A. Vallejo, and F. Guhl, "Seroprevalence of Trypanosoma cruzi in dogs from two endemic areas of Colombia," Revista Médica, vol. 16, no. 1, pp. 11-18, 2008.

[10] S. C. Barr, R. A. Holmes, and T. R. Klei, "Electrocardiographic and echocardiographic features of trypanosomiasis in dogs inoculated with North American Trypanosoma cruzi isolates," American Journal of Veterinary Research, vol. 53, no. 4, pp. 521527, 1992.

[11] M. de Lana, E. Chiari, and W. L. Tafuri, "Experimental Chagas' disease in dogs," Memorias do Instituto Oswaldo Cruz, vol. 87, no. 1, pp. 59-71, 1992.

[12] M. T. Bahia, W. L. Tafuri, M. V. Caliari et al., "Comparison of Trypanosoma cruzi infection in dogs inoculated with blood or metacyclic trypomastigotes of Berenice- 62 and Berenice-78 
strains via intraperitoneal and conjunctival routes," Revista da Sociedade Brasileira de Medicina Tropical, vol. 35, no. 4, pp. 339345, 2002.

[13] M. V. Caliari, M. de Lana, R. A. Cajá et al., "Immunhistochemical studies in acute and chronic canine chagasic cardiomyopathy," Virchows Archiv, vol. 441, no. 1, pp. 69-76, 2002.

[14] M. V. Caliari, R. D. P. Machado, M. de Lana et al., "Quantitative analysis of cardiac lesions in chronic canine chagasic cardiomyopathy," Revista do Instituto de Medicina Tropical de Sao Paulo, vol. 44, no. 5, pp. 273-278, 2002.

[15] V. M. Montenegro, M. Jiménez, J. C. Dias, and R. Zeledón, "Chagas disease in dogs from endemic areas of Costa Rica," Memorias do Instituto Oswaldo Cruz, vol. 97, no. 4, pp. 491-494, 2002.

[16] P. M. M. Guedes, V. M. Veloso, K. J. Gollob et al., "IgG isotype profile is correlated with cardiomegaly in Beagle dogs infected with distinct Trypanosoma cruzi strains," Veterinary Immunology and Immunopathology, vol. 124, no. 1-2, pp. 163$168,2008$.

[17] I. A. Quijano-Hernandez, M. E. Bolio-González, J. C. Rodríguez-Buenfil, M. J. Ramirez-Sierra, and E. Dumonteil, "Therapeutic DNA vaccine against Trypanosoma cruzi infection in dogs: a pilot clinical trial," Annals of the New York Academy of Sciences, vol. 1149, pp. 343-346, 2008.

[18] P. M. M. Guedes, V. M. Veloso, L. C. C. Afonso et al., "Development of chronic cardiomyopathy in canine Chagas disease correlates with high IFN- $\gamma$, TNF- $\alpha$, and low IL-10 production during the acute infection phase," Veterinary Immunology and Immunopathology, vol. 130, no. 1-2, pp. 43-52, 2009.

[19] J. E. Aparicio-Burgos, L. Ochoa-García, J. A. Zepeda-Escobar et al., "Testing the efficacy of a multi-component DNAprime/DNA-boost vaccine against Trypanosoma cruzi infection in dogs," PLoS Neglected Tropical Diseases, vol. 5, no. 5, Article ID e1050, 2011.

[20] J. Mota-Sánchez, "Vacunas de ADN: inducción de la respuesta inmunitaria," Salud Pública de México, vol. 51, suplement 3, pp. S463-S469, 2009.

[21] M. Arce-Fonseca, A. Ramos-Ligonio, A. López-Monteón, B. Salgado- Jiménez, P. Talamás-Rohana, and J. L. Rosales-Encina, "A DNA vaccine encoding for TcSSP4 induces protection against acute and chronic infection in experimental Chagas disease," International Journal of Biological Sciences, vol. 7, no. 9, pp. 1230-1238, 2011.

[22] B. Salgado-Jiménez, M. Arce-Fonseca, L. Baylón-Pacheco, P. Talamás-Rohana, and J. L. Rosales-Encina, "Differential immune response in mice immunized with the $\mathrm{A}, \mathrm{R}$ or $\mathrm{C}$ domain from TcSP protein of Trypanosoma cruzi or with the coding DNAs," Parasite Immunology, vol. 35, no. 1, pp. 32-41, 2013.

[23] WHO Technical Report Series, Control of Chagas Disease. Second Report of the WHO Expert Committee, pp. 1-109, WHO, Geneva, Switzerland, 2002.

[24] O. Rodríguez-Morales, M. M. Pérez-Leyva, M. A. BallinasVerdugo et al., "Plasmid DNA immunization with Trypanosoma cruzi genes induces cardiac and clinical protection against Chagas disease in the canine model," Veterinary Research, vol. 43, no. 1, p. 79, 2012.

[25] O. Rodríguez-Morales, M. A. Ballinas-Verdugo, R. AlejandreAguilar, P. A. Reyes, and M. Arce-Fonseca, "Trypanosoma cruzi connatal transmission in dogs with chagas disease: experimental case report," Vector-Borne and Zoonotic Diseases, vol. 11, no. 10, pp. 1365-1370, 2011.
[26] Norma Oficial Mexicana NOM-0062-ZOO-1999, Especificaciones Técnicas para el Cuidado y Uso de Animales de Laboratorio, pp. 1-58, Diario Oficial de la Federación, Mexico City, Mexico, 1999.

[27] Norma Oficial Mexicana NOM-033-ZOO-1995, Sacrificio humanitario de los animales domésticos y silvestres, pp. 1-17, Diario Oficial de la Federación, Mexico City, Mexico, 1995.

[28] V. M. Monteón, J. Furuzawa-Carballeda, R. Alejandre-Aguilar, A. Aranda-Fraustro, J. L. Rosales-Encina, and P. A. Reyes, "American trypanosomosis: in situ and generalized features of parasitism and inflammation kinetics in a murine model," Experimental Parasitology, vol. 83, no. 3, pp. 267-274, 1996.

[29] L. P. Tilley, Essentials of Canine and Feline Electrocardiography: Interpretation and Treatment, Lippincott Wiliams \& Wilkins, Baltimore, Md, USA, 1992.

[30] L. P. Tilley LP and N. L. Burtnick, ECG Electrocardiography for the Small Animal Practitioner, Teton New Media, Jackson, Wyo, USA, 1999.

[31] P. M. M. Guedes, V. M. Veloso, M. V. Caliari et al., "Trypanosoma cruzi high infectivity in vitro is related to cardiac lesions during long-term infection in Beagle dogs," Memorias do Instituto Oswaldo Cruz, vol. 102, no. 2, pp. 141-147, 2007.

[32] S. C. Barr, S. P. Schmidt, C. C. Brown, and T. R. Klei, "Pathologic features of dogs inoculated with North American Trypanosoma cruzi isolates," American Journal of Veterinary Research, vol. 52, no. 12, pp. 2033-2039, 1991.

[33] B. Espinoza, T. Rico, S. Sosa et al., "Mexican Trypanosoma cruzi T. cruzi i strains with different degrees of virulence induce diverse humoral and cellular immune responses in a murine experimental infection model," Journal of Biomedicine and Biotechnology, vol. 2010, Article ID 890672, 10 pages, 2010.

[34] B. Espinoza, N. Solorzano-Domínguez, A. Vizcaino-Castillo, I. Martínez, A. L. Elias-López, and J. A. Rodríguez-Martínez, "Gastrointestinal infection with Mexican TcI Trypanosoma cruzi strains: different degrees of colonization and diverse immune responses," International Journal of Biological Sciences, vol. 7, no. 9, pp. 1357-1370, 2011.

[35] M. Arce-Fonseca, M. A. Ballinas-Verdugo, E. R. Abreu-Zenteno et al., "Specific humoral and cellular immunity induced by Trypanosoma cruzi DNA immunization in a canine model," Veterinary Research, vol. 44, no. 1, p. 15, 2013.

[36] I. A. Quijano-Hernández, A. Castro-Barcena, E. AparicioBurgos et al., "Evaluation of clinical and immunopathological features of different infective doses of Trypanosoma cruzi in dogs during the acute phase," The Scientific World Journal, vol. 2012, Article ID 635169, 6 pages, 2012.

[37] N. Añez, G. Crisante, and H. Parada, "Nuevos casos agudos de enfermedad de Chagas en el Occidente de Venezuela," Salus Revista de la Facultad de Ciencias de la Salud, Universidad de Carabobo, vol. 11, Suplement 1, pp. 87-90, 2007.

[38] E. M. M. Machado, A. J. Fernandes, S. M. F. Murta et al., "A study of experimental reinfection by Trypanosoma cruzi in dogs," The American Journal of Tropical Medicine and Hygiene, vol. 65, no. 6, pp. 958-965, 2001.

[39] N. Garg and R. L. Tarleton, "Genetic immunization elicits antigen-specific protective immune responses and decreases disease severity in Trypanosoma cruzi infection," Infection and Immunity, vol. 70, no. 10, pp. 5547-5555, 2002. 


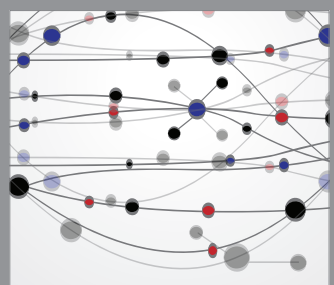

The Scientific World Journal
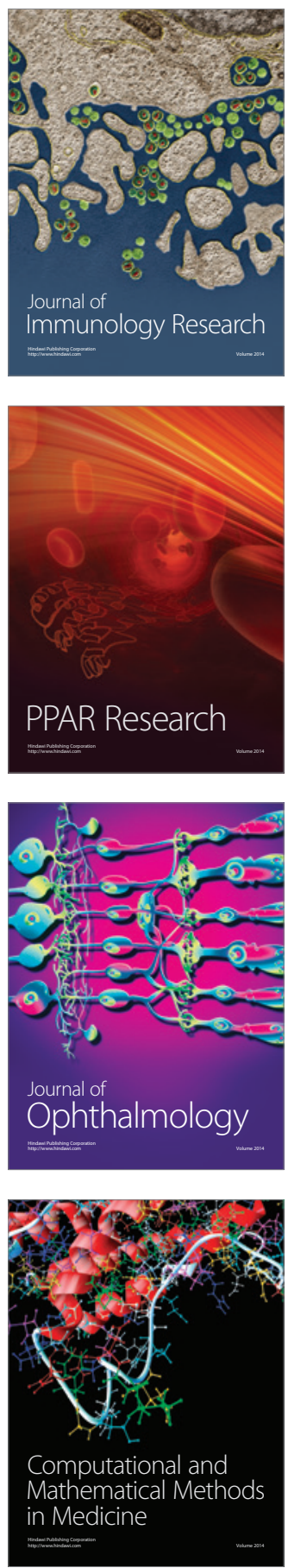

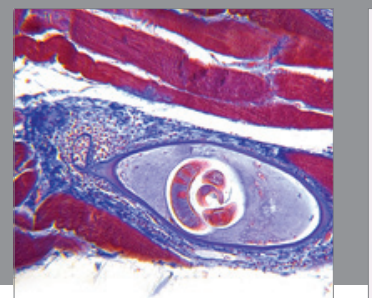

Gastroenterology

Research and Practice
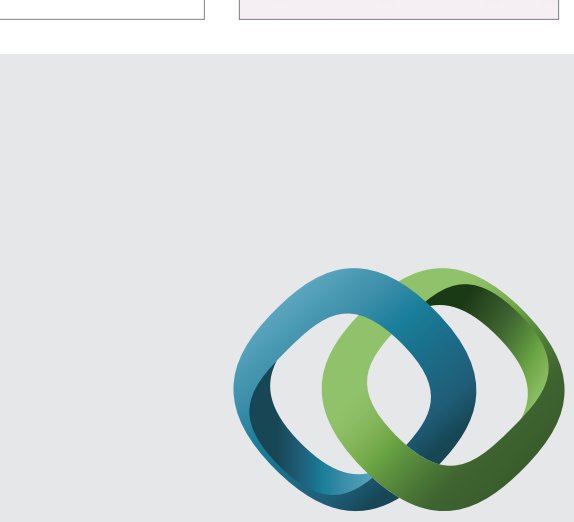

\section{Hindawi}

Submit your manuscripts at

http://www.hindawi.com
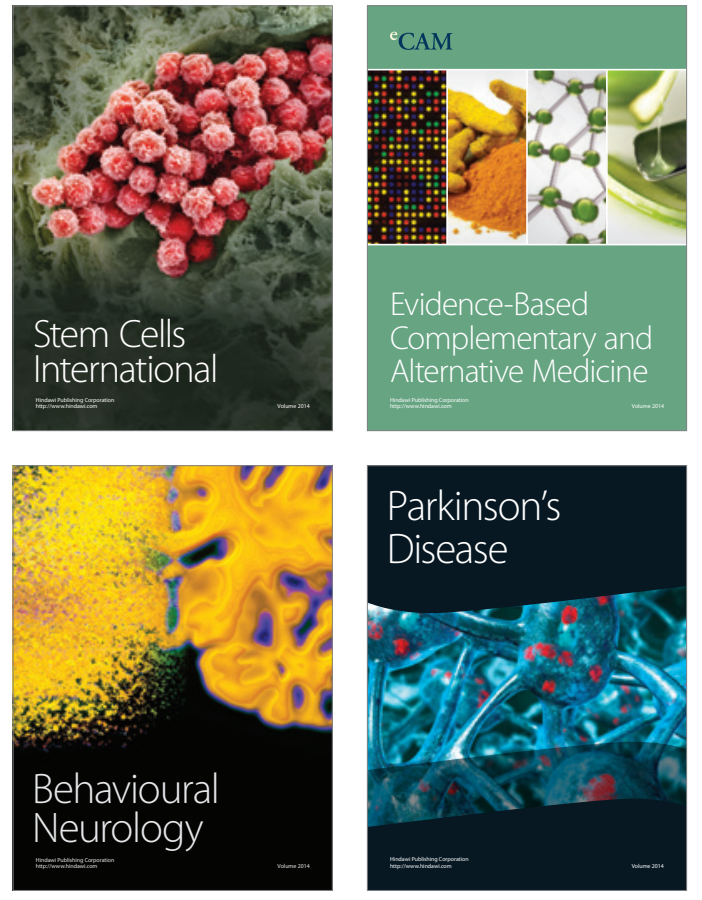
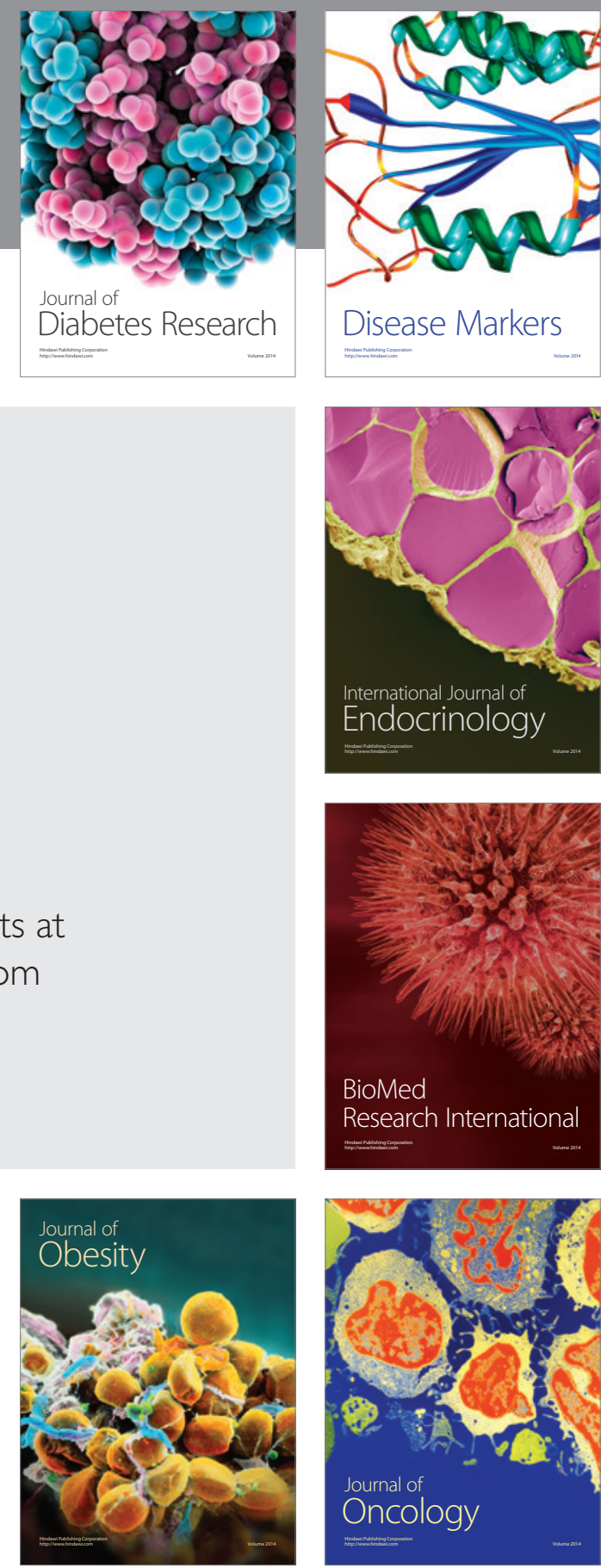

Disease Markers
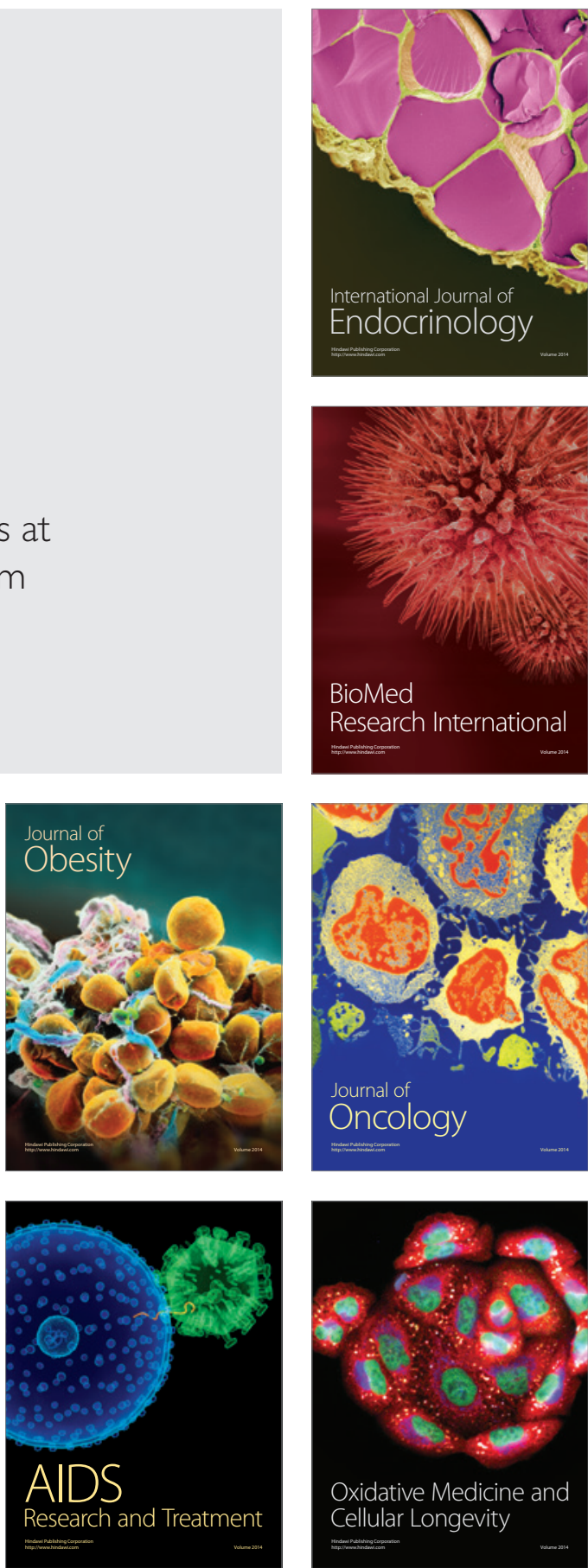